\title{
Family specific genetic predisposition to breast cancer: results from Tunisian whole exome sequenced breast cancer cases
}

\author{
Yosr Hamdi ${ }^{*}$ (D), Maroua Boujemaa ${ }^{1}$, Mariem Ben Rekaya ${ }^{1}$, Cherif Ben Hamda ${ }^{2,3}$, Najah Mighri', \\ Houda El Benna ${ }^{4}$, Nesrine Mejri ${ }^{4}$, Soumaya Labidi ${ }^{4}$, Nouha Daoud $^{4}$, Chokri Naouali ${ }^{1}$, Olfa Messaoud', \\ Mariem Chargui ${ }^{1}$, Kais Ghedira², Mohamed Samir Boubaker ${ }^{1}$, Ridha Mrad ${ }^{5}$, Hamouda Boussen ${ }^{4}$, \\ Sonia Abdelhak ${ }^{1}$ on behalf of the PEC Consortium ${ }^{4}$
}

\begin{abstract}
Background: A family history of breast cancer has long been thought to indicate the presence of inherited genetic events that predispose to this disease. In North Africa, many specific epidemio-genetic characteristics have been observed in breast cancer families when compared to Western populations. Despite these specificities, the majority of breast cancer genetics studies performed in North Africa remain restricted to the investigation of the BRCA1 and BRCA2 genes. Thus, comprehensive data at a whole exome or whole genome level from local patients are lacking.

Methods: A whole exome sequencing (WES) of seven breast cancer Tunisian families have been performed using a family-based approach. We focused our analysis on BC-TN-F001 family that included two affected members that have been sequenced using WES. Relevant variants identified in BC-TN-F001 have been confirmed using Sanger sequencing. Then, we conducted an integrative analysis by combining our results with those from other WES studies in order to figure out the genetic transmission model of the newly identified genes. Biological network construction and protein-protein interactions analyses have been performed to decipher the molecular mechanisms likely accounting for the role of these genes in breast cancer risk.
\end{abstract}

Results: Sequencing, filtering strategies, and validation analysis have been achieved. For BC-TN-F001, no deleterious mutations have been identified on known breast cancer genes. However, 373 heterozygous, exonic and rare variants have been identified on other candidate genes. After applying several filters, 12 relevant high-risk variants have been selected. Our results showed that these variants seem to be inherited in a family specific model. This hypothesis has been confirmed following a thorough analysis of the reported WES studies. Enriched biological process and proteinprotein interaction networks resulted in the identification of four novel breast cancer candidate genes namely MMS19, DNAH3, POLK and KATB6.

Conclusions: In this first WES application on Tunisian breast cancer patients, we highlighted the impact of next generation sequencing technologies in the identification of novel breast cancer candidate genes which may bring new insights into the biological mechanisms of breast carcinogenesis. Our findings showed that the breast cancer predisposition in non-BRCA families may be ethnic and/or family specific.

Keywords: Breast cancer, Exome sequencing, Family specific predisposition, Non BRCA Tunisian families

\footnotetext{
*Correspondence: yosr.hamdi.82@gmail.com

1 Laboratory of Biomedical Genomics and Oncogenetics, LR16IPT05,

Institut Pasteur de Tunis, University of Tunis El Manar, 13, Place Pasteur-BP

74, 1002 Tunis, Tunisia

Full list of author information is available at the end of the article
} 


\section{Background}

A range of genetic and non-genetic risk factors contribute to the development of breast cancer [1]. So far, several genetic variants of high, moderate and low penetrance have been identified as impacting on breast cancer risk using familial linkage, DNA resequencing and genome wide association analysis, respectively [2]. The identification of additional breast cancer associated genes is crucial to explain the missing breast cancer heritability. Recent studies showed that breast cancer susceptibility may be explained by a polygenic risk model of inheritance in which a large number of common SNPs contribute multiplicatively towards risk [3]. With the introduction of next generation sequencing (NGS) technologies [4, 5] many studies suggested that a large rate of the remaining breast cancer heritability can be attributed to new rare risk alleles that segregate in an autosomal-dominant pattern of inheritance.

To date, two different whole exome sequencing study designs are used: case/control association studies and the family-based approach. The case/control design is considered as the major promising tool to detect significant associations between genetic variations and breast cancer disease [6]. However, due to the extreme rarity of certain variants, this approach requires large-size cohorts to confirm the association between these variants and breast cancer risk. The second WES design is the familybased approach [7] where breast cancer family members are exome-sequenced and the shared variants between affected individuals presumably include the familial breast cancer risk allele. Thus, focusing on the family segregation of relevant variants is expected to better detect novel susceptibility variants than the screening of pooled unrelated cases and controls.

Several WES studies have been performed on hereditary breast cancer [7, 8]. Almost, 108 breast cancer families have been whole exome sequenced using the family-based approach and reported many relevant variants present in related affected individuals and absent in unaffected ones. So far, five new genes have been identified by WES as associated with breast cancer risk, among them four genes identified using the family-based approach, namely: XRCC2 [9], MAPKAP1 [10], FANCM [11] and RINT1 [12] while only one gene, REQCL, was identified using the case/control approach [13]. Mutations on known breast cancer susceptibility genes were reported in only four families [10-14].

In Tunisia, breast cancer is the most common and the most deadly form of cancer among females [15]. Several epidemiological, genetic and clinical breast cancer characteristics have been observed to be unique to Tunisian and North African population. Indeed, breast cancer shows a lower incidence rate but a younger age of disease onset, when compared to Western populations, with a relative high frequency of the aggressive breast cancer forms such as inflammatory and triple negative breast cancers [16]. Thus, a genetic predisposition specific to this ethnic group is plausible, $[8,17,18]$. Moreover, it is possible that breast cancer risk variants are so rare that they are "family specific" meaning that a genetic predisposition can be detected within a disease-prone family, but not necessarily shared with other genetically unrelated families with the same disease [19-21].

So far, genetic studies performed on Tunisian breast cancer patients mostly focused on the BRCA genes using the traditional Sanger technique. Therefore, the use of next generation sequencing technologies in the genetic investigation of these under-exploited populations may help identifying novel breast cancer risk allele and explain the remaining unresolved breast cancer genetic heritability.

In the present study, we performed a whole exome sequencing of seven BRCAx breast cancer Tunisian families with strong family history in order to identify genetic variations that may be associated with breast cancer risk. Using the family-based approach, we focused our analysis on a non BRCA family by sequencing two out of three affected sisters. After comparing our results to those identified in previous WES studies and by performing biological network analysis, we identified a set of novel breast cancer candidate genes that seems to be inherited in a family specific manner.

\section{Methods \\ Patients}

Seven Tunisian breast cancer families were selected for WES based on the following criteria: (1) Presence of at least three related first or second-degree breast cancer cases; (2) Breast cancer in young patients aged less than 35 years, (3) Presence of at least two cases of breast or ovarian cancer, regardless of age, and at least one case of pancreatic cancer or prostate cancer in a related first or second degree patient. Blood samples have been collected from the affected family members and have been sampled in the Medical oncology department, Abderrahman Mami Hospital, Ariana, Tunisia. Written informed consents were obtained from all participants. Ethical approval according to the Declaration of Helsinki Principles was obtained from the biomedical ethics committee of Institut Pasteur de Tunis (2017/16/E/Hôpital a-m/V1).

Two out of three affected sisters from BC-TN-F001 have been whole exome sequenced. The proband was diagnosed with a primary breast cancer at age 43 and contralateral invasive ductal breast carcinoma at age 48. The second family member involved in this study was diagnosed with an invasive breast cancer at age 56 . 
Phenotypic characteristics of the affected family members are described in Table 1.

\section{Whole exome sequencing and data analysis}

For each participant, total genomic DNA was isolated from peripheral blood using the salting out method or the DNeasy blood Kit from Qiagen according to the manufacturer's instructions. DNA purity and concentration were measured using a NanoDrop ${ }^{\mathrm{TM}}$ spectrophotometer.

Samples were prepared according to Agilent's SureSelect Protocol version 1.2 and enrichment was carried out according to Agilent SureSelect protocols. Enriched samples were sequenced on the Illumina HiSeq 2000 platform using TruSeq v3 chemistry with paired-end $(2 \times 100 \mathrm{pb})$.

Exome DNA sequences were mapped to their location in the build of the human genome (hg19/b37) using the Burrows-Wheeler Aligner (BWA) package. The subsequent SAM files were converted to BAM files using Samtools. Duplicate reads were removed using Picard. GATK was then used to recalibrate the base quality scores as well as for SNP and short INDEL calling. Annotation and prioritization of potential disease-causing variants were performed using VarAFT (Variant Annotation and Filtering Tool) (http://varaft.eu). To annotate variants, VarAFT uses ANNOVAR, a command line tool. INDELs and SNPs annotated were filtered according to several criteria: (1) considering breast cancer as autosomal dominant disease and removing variants that were found in a homozygous state, (2) variants identified as intronic, intergenic, and none coding or synonymous were discarded, (3) assuming that causal variants are rare, we removed all variants with an allele frequency $>1 \%$ either in Exac [22], 1000 genomes [23] or ESP6500 (http://evs. gs.washington.edu/EVS/), (4) benign or tolerated variants, according to different in silico prediction tools were also removed. Finally, significant candidate variants were obtained after filtering against their phenotypic relevance.

\section{Sanger sequencing}

The Sanger sequencing technique was first used to test the $B R C A$ status of affected family members, then to validate the identified variants resulting from the whole exome sequencing. PCR reactions were performed on genomic DNA (gDNA), following standard protocols, followed by Sanger sequencing using an automated sequencer (ABI 3500; Applied Biosystems, Foster City, CA) using a cycle sequencing reaction kit (Big Dye Terminator kit, Applied Biosystems). Data were analyzed using BioEdit Sequence Alignment Editor Version 7.2.5.

\section{In silico prediction tools}

We selected four in silico prediction tools to assess the functional effects of the candidate variants: Sorting Intolerant From Tolerant (SIFT) (http://sift.jcvi.org/) to examine the degree of conservation for amino acid residues across species and to find changes in protein structure and function; PolyPhen-2 (http://genetics.bwh. harvard.edu/pph2/) and Mutation Taster (http://www. mutationtaster.org/) to assess the impact of mutations on protein function and to look at effects on splicing or mRNA expression and Align GVGD (http://agvgd.iarc.fr) that classifies missense variants in a query sequence into seven grades, from the most deleterious $\mathrm{C} 65$ to the least deleterious $\mathrm{C} 0$, with the intermediate grades $\mathrm{C} 15, \mathrm{C} 25$, C35, C45 and C55 [24]. The program is based on Grantham calculation, a combination of Grantham Variation (GV) which measures the amount of observed biochemical evolutionary variation at a specific position of the alignment, and Grantham Deviation (GD) which measures the biochemical difference between the missense residue and the range of variation observed at this position in the alignment.

Table 1 Epidemiological and clinical data of affected family members

\begin{tabular}{|c|c|c|c|c|c|c|c|c|}
\hline Family Member & Diagnosis age & $\begin{array}{l}\text { Histological } \\
\text { subtype }\end{array}$ & SBR grade & $\begin{array}{l}\text { Tumor } \\
\text { size } \\
(\mathrm{mm})\end{array}$ & $\begin{array}{l}\text { Hormone } \\
\text { receptors } \\
\text { status }\end{array}$ & HER2 status & $\begin{array}{l}\text { Disease } \\
\text { evolution }\end{array}$ & Medical history \\
\hline BC-TN-F001-1 & 43 & $\begin{array}{l}\text { Invasive ductal } \\
\text { carcinoma }\end{array}$ & $\|$ & 22 & $\mathrm{ER}+/ \mathrm{PR}+$ & ND & $\begin{array}{l}\text { CBC within } \\
5 \text { years, grade III } \\
\text { triple negative } \\
\text { carcinoma }\end{array}$ & 3 miscarriages \\
\hline BC-TN-F001-2 & 56 & $\begin{array}{l}\text { Invasive ductal } \\
\text { carcinoma }\end{array}$ & ND & ND & $\mathrm{ER}+/ \mathrm{PR}+$ & ND & In remission & No medical history \\
\hline BC-TN-F001-3 & 47 & $\begin{array}{l}\text { Bifocal invasive } \\
\text { ductal carci- } \\
\text { noma }\end{array}$ & I & 7 & $\mathrm{ER}+/ \mathrm{PR}+$ & HER2- & In remission & $\begin{array}{l}\text { Primary infertility } \\
\text { (IVF) }\end{array}$ \\
\hline
\end{tabular}


Functional annotation and biological network construction To discern the implication of the candidate breast cancer genes, several bioinformatics tools have been used to explore their biological pathways and the possible protein-protein interactions.

We first performed a functional analysis using the EnrichR platform [25], a bioinformatics web-based tool that includes more than 60 gene-set libraries, such as Gene ontology [26], KEGG, Wikipathways, as well as Jensendiseases. The selection criteria for significantly enriched pathways and ontology term were a $p$ value less than 0.05 (Additional file 1: Table S1).

For a better visualization and interpretation of the biological processes associated with selected breast cancer candidate genes and their upstream regulator, we used ClueGO [27], a user friendly Cytoscape plug-into analyze interrelations of terms and functional groups in biological networks [28]. In brief, we used enrichment (right-sided) hyper-geometric distribution tests, with a $\mathrm{p}$ value significance level $\leq 0.05$, followed by the Bonferroni adjustment for the terms and the groups with Kappa-statistics score threshold set to 0.5, and leading term groups were selected based on the highest significance.

Protein-protein interaction network including physical and functional association across our set of genes was sorted out using string db 10.0 [29] with confidence score 0.4 .

\section{Results}

Eight affected individuals from seven BRCAx Tunisian families at high risk of breast cancer were analyzed using whole exome sequencing. Results including number of reads, sample coverage and sequencing depth of the whole exome sequenced patients have been summarized in Additional file 1: Table S2.

We focused our current analysis on the first $B R C A$ negative family; BC-TN-F001 (Fig. 1). Two out of three affected family members have been selected for whole exome sequencing.

\section{Analysis of variants located on the known breast cancer susceptibility genes}

Before applying the filter, steps described in the methods section, we first investigated the following 29 genes known to be associated with hereditary breast and ovarian cancer: ATM, BARD1, BRCA1, BRCA2, BLM, BRIP1, CDH1, CHEK2, FAM175A, FANCC, FANCM, MAPKAP1, MLH1, MRE11A, MSH2, NBN, NF1, PALB2, PMS2, PTEN, RAD50, RAD51B, RAD51C, RAD51D, RECQL, RINT1, STK11, TP53 and XRCC2 (Table 2). 59 shared heterozygous variants have been identified on these genes of which, 51 (86.4\%) common noncoding variants, five exonic variants and 3 splicing SNPs. The exonic variations include a $B R C A 2$ rare variant (rs4987047, MAF $=0.0089)$, three common exonic polymorphisms on BARD1 (rs2070094, rs2229571 and rs1048108), and one variant on MAPKAP1 (rs1201689). None of the heterozygous variants that have been found on BRCA1, BLM, FAM175A, FANCM, PTEN, RAD50,
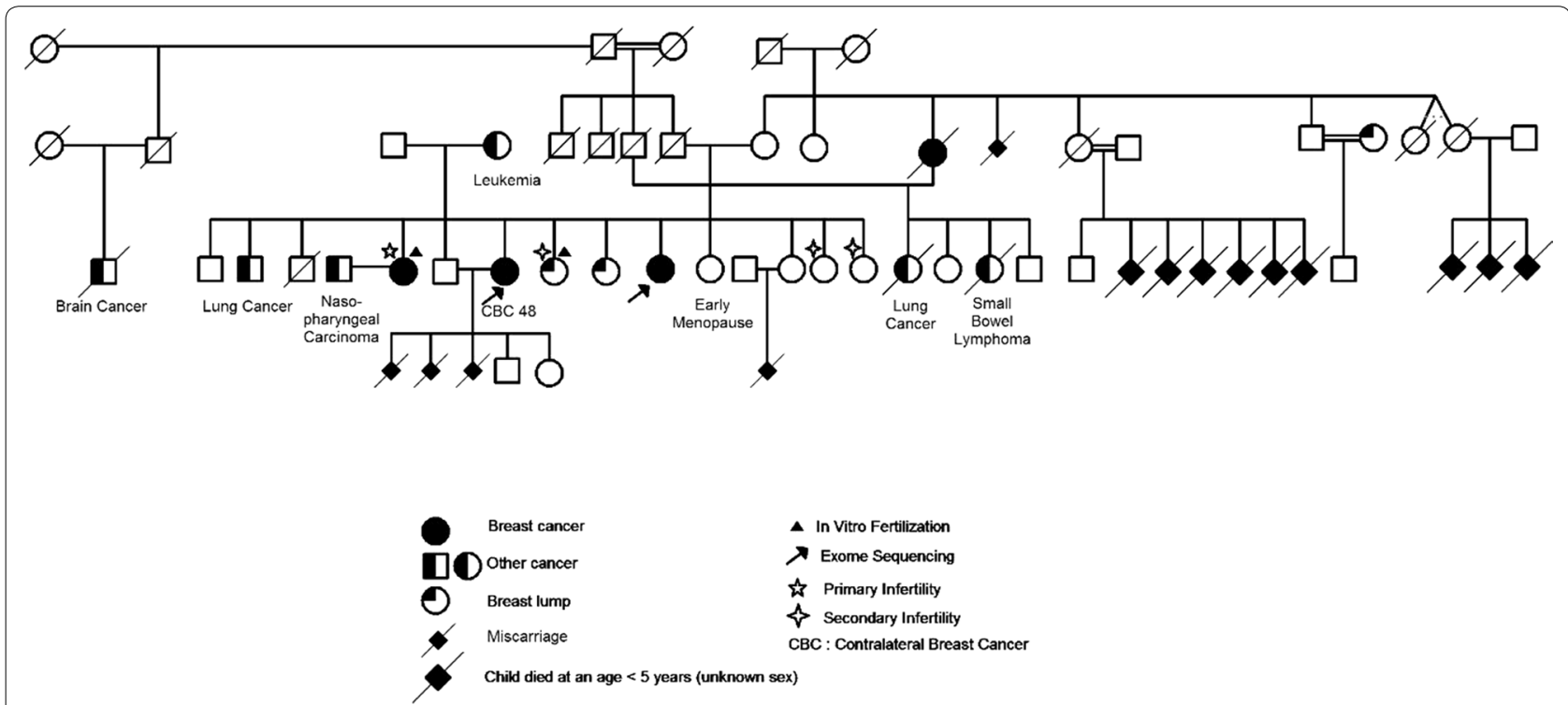

Fig. 1 The familial pedigree of the breast cancer whole exome sequenced family 
Table 2 Variants on hereditary breast and ovarian cancer genes shared by the two sequenced family members

\begin{tabular}{|c|c|c|c|c|c|c|}
\hline Genes & Position & Variant ID & Sequence variation & $\begin{array}{l}\text { Frequency (1000 } \\
\text { genomes) }\end{array}$ & Localization & ClinVar \\
\hline \multirow[t]{3}{*}{ ATM } & 108137775 & rs642496 & c. $2467-123 T>A$ & 0.681909 & Intronic & - \\
\hline & 108225661 & rs664143 & c. $640+30986 T>C$ & 0.628195 & Intronic & - \\
\hline & 10815,707 & rs3218681 & c.3403-15_3403-14insA & 0.542133 & Intronic & Benign \\
\hline \multirow[t]{8}{*}{ BARD1 } & 215632255 & rs2070094 & c. $1462 \mathrm{G}>\mathrm{A}$ & 0.366214 & Exonic & Likely benign \\
\hline & 215645464 & rs2229571 & c. $1077 G>C$ & 0.459265 & Exonic & Likely benign \\
\hline & 215674224 & rs1048108 & $c .70 \mathrm{C}>\mathrm{T}$ & 0.33127 & Exonic & Likely benign \\
\hline & 215632155 & rs5031009 & c. $1568+51 A>G$ & 0.366214 & Intronic & - \\
\hline & 215632126 & rs398048293 & c.1511+78_1511+79delAA & 0.366214 & Intronic & - \\
\hline & 215634055 & rs6704780 & c.1315-19G>A & 0.365216 & Intronic & Benign \\
\hline & 21532192 & rs5031011 & c. $1568+14 C>T$ & 0.352236 & Intronic & Likely benign \\
\hline & 215595645 & rs16852600 & c. $1904-413 G>A$ & 0.275359 & Intronic & - \\
\hline$B L M$ & \multicolumn{6}{|c|}{ No detected variants } \\
\hline$B R C A 1$ & \multicolumn{6}{|c|}{ No detected variants } \\
\hline BRCA2 & 32953529 & rs4987047 & c. $8830 \mathrm{~A}>\mathrm{T}$ & 0.00898562 & Exonic & Benign \\
\hline BRIP1 & \multicolumn{6}{|c|}{ No detected variants } \\
\hline \multirow[t]{3}{*}{$\mathrm{CDH} 1$} & 68857277 & rs201760019 & C. $1754-25 C>A$ & 0.000599042 & Intronic & - \\
\hline & 68857544 & rs34939176 & c.1981+17_1981+18insA & 0.0459265 & Intronic & Benign \\
\hline & 68868148 & rs 140240766 & $c^{*} 746 C>A$ & 0.000599042 & UTR3 & Likely benign \\
\hline CHEK2 & 29137944 & rs2236142 & c. $-194 C>G$ & 0.560304 & Upstream & - \\
\hline FAM175A & \multicolumn{6}{|c|}{ No detected variants } \\
\hline \multirow[t]{3}{*}{ FANCC } & 9,873957 & rs4647534 & C. $1155-38 \mathrm{~T}>\mathrm{C}$ & 0.541334 & Intronic & Benign \\
\hline & 97873435 & rs2404457 & c. $1329+310 C>T$ & 0.411142 & UTR3 & - \\
\hline & 97888730 & rs4647512 & $\mathrm{c} .896+81 \mathrm{G}>\mathrm{A}$ & 0.0313498 & Intronic & - \\
\hline FANCM & \multicolumn{6}{|c|}{ No detected variants } \\
\hline \multirow[t]{4}{*}{ MAPKAP1 } & 128321827 & rs146481224 & $c .848+85 T>A$ & 0.0163738 & Intronic & - \\
\hline & 42103822 & rs1197672 & c. $328-333 C>T$ & 0.239816 & Intronic & - \\
\hline & 42105918 & rs1201689 & $c .937 C>G$ & 0.305112 & Exonic & - \\
\hline & 42111933 & rs890497 & c. $2499+85 G>A$ & 0.0884585 & Intronic & - \\
\hline MLH1 & 37070437 & rs41562513 & c. $1558+14 G>A$ & 0.0501198 & Intronic & Benign \\
\hline \multirow[t]{6}{*}{ MRE11A } & 94179125 & rs1014666 & c. $1784-69 A>G$ & 0.517173 & Intronic & - \\
\hline & 94212048 & rs535801 & $c .403-6 \mathrm{G}>\mathrm{A}$ & 0.313099 & Splicing & Benign \\
\hline & 94197568 & rs640627 & c. $1099-163 G>A$ & 0.314896 & Intronic & - \\
\hline & 94225807 & rs496797 & c. $20+141 \mathrm{G}>\mathrm{A}$ & 0.552915 & Splicing & - \\
\hline & 94225920 & rs497763 & c. $20+28 \mathrm{G}>\mathrm{A}$ & 0.457268 & Intronic & Benign \\
\hline & 94212154 & rs680695 & $c .403-112 T>C$ & 0.313099 & Intronic & - \\
\hline \multirow[t]{5}{*}{ MSH2 } & 47656801 & rs2347794 & c. $1077-80 G>A$ & 0.59365 & Intronic & Benign \\
\hline & 47630550 & rs2303426 & $c .211+9 C>A$ & 0.628395 & Intronic & Benign \\
\hline & 47693959 & rs3732183 & c. $1661+12 \mathrm{G}>\mathrm{A}$ & 0.483427 & Intronic & Benign \\
\hline & 47693706 & rs3732182 & c. $1511-91 G>T$ & 0.483027 & Intronic & Benign \\
\hline & 47739551 & rs2303424 & c. $2744 A>G$ & 0.527955 & Intergenic & - \\
\hline$N B N$ & 90983317 & rs 104895036 & $c .456+84 G>C$ & 0.00139776 & Intronic & - \\
\hline$N F 1$ & 29685905 & rs34513299 & c. $8051-82 A>G$ & 0.00199681 & Intronic & - \\
\hline \multirow[t]{2}{*}{ PALB2 } & 23640467 & rs249954 & c. $2586+58 C>T$ & 0.35004 & Intronic & Benign \\
\hline & 23652525 & rs8053188 & c. $-339 C>T$ & 0.0662939 & UTR5 & Benign \\
\hline PMS2 & 6037058 & rs549498051 & c.706-5delT & 0.453075 & Splicing & Benign \\
\hline PTEN & \multicolumn{6}{|c|}{ No detected variants } \\
\hline
\end{tabular}


Table 2 (continued)

\begin{tabular}{|c|c|c|c|c|c|c|}
\hline Genes & Position & Variant ID & Sequence variation & $\begin{array}{l}\text { Frequency (1000 } \\
\text { genomes) }\end{array}$ & Localization & ClinVar \\
\hline \multirow[t]{4}{*}{ RAD50 } & 131927748 & rs10520116 & C. $1793+22 T>C$ & 0.0129792 & Intronic & - \\
\hline & 131944964 & rs2066742 & c.2923-11_2923-10insT & 0.0734824 & Intronic & Likely benign \\
\hline & 131928652 & rs2706366 & c. $1793+926 \mathrm{~A}>\mathrm{G}$ & 0.123003 & Intronic & - \\
\hline & 131892979 & rs4526098 & c. $-38 \mathrm{~A}>\mathrm{G}$ & 0.92492 & UTR5 & Benign \\
\hline \multirow[t]{9}{*}{$R A D 51 B$} & 68290372 & rs17783124 & $\mathrm{c} .84+28 \mathrm{~T}>\mathrm{G}$ & 0.250399 & Intronic & - \\
\hline & 68290464 & rs 28623567 & $c .84+120 G>A$ & 0.2498 & Intronic & - \\
\hline & 68937054 & rs142879847 & c. $1036+2087 A>G$ & 0.00798722 & Intronic & - \\
\hline & 68758575 & rs10129646 & c. $757-26 \mathrm{~T}>\mathrm{C}$ & 0.138379 & Intronic & - \\
\hline & 68301767 & rs34564590 & c.199-29_199-28insA & 0.319489 & Intronic & - \\
\hline & 68290426 & rs28604984 & $\mathrm{c} .84+82 \mathrm{~T}>\mathrm{C}$ & 0.2498 & Intronic & - \\
\hline & 68934860 & rs34436700 & c. $958-29 A>G$ & 0.00778754 & Intronic & Likely benign \\
\hline & 69117512 & rs8023214 & c. $1037-32142 T>C$ & 0.528554 & Intergenic & - \\
\hline & 69117387 & rs8021657 & c. $1037-32267 A>G$ & 0.527556 & Intergenic & - \\
\hline \multirow[t]{2}{*}{ RAD51C } & 56798207 & rs28363318 & c. $904+34 \mathrm{~T}>\mathrm{C}$ & 0.205272 & Intronic & - \\
\hline & 56769979 & rs12946397 & c. $-681 \mathrm{G}>\mathrm{A}$ & 0.158347 & UTR5 & Likely benign \\
\hline RAD51D & \multicolumn{6}{|c|}{ No detected variants } \\
\hline \multirow[t]{4}{*}{ RECQL } & 21629993 & rs397718052 & c.868-68_868-67insG & 0.488818 & Intronic & - \\
\hline & 21628320 & rs10841831 & c. $1216+82 \mathrm{G}>\mathrm{A}$ & 0.486821 & Intronic & - \\
\hline & 21628791 & rs3752648 & c. $950-33 \mathrm{~A}>\mathrm{G}$ & 0.48742 & Intronic & - \\
\hline & 21628336 & rs10841832 & C. $1216+66 C>T$ & 0.486821 & Intronic & - \\
\hline RINT1 & \multicolumn{6}{|c|}{ No detected variants } \\
\hline STK11 & \multicolumn{6}{|c|}{ No detected variants } \\
\hline TP53 & \multicolumn{6}{|c|}{ No detected variants } \\
\hline$X R C C 2$ & \multicolumn{6}{|c|}{ No detected variants } \\
\hline
\end{tabular}

RINT1, STK11, TP53 and XRCC2 were shared between the two sequenced family members.

Based on breast cancer information core (BIC) and ClinVar databases, none of the 59 variants identified on these classical breast and ovarian cancer genes was classified as pathogenic. Thus, we suggested that breast cancer genetic predisposition in this family might be due to new variants on novel breast cancer candidate genes.

\section{Identification of novel candidate variants}

A total of 32,212 heterozygous variants shared by both cases have been identified (Fig. 2). Among them, 4593 heterozygous, exonic, splicing and non-synonymous SNPs were called. Variants with MAF $>1 \%$ have been excluded. Therefore, 373 rare variations have been selected for further investigations including 39 variations that have not been previously reported. In fact, as the Tunisian population is not represented in public databases, reported variants have not been excluded.

In order to select the most relevant SNPs, SIFT (score $<0.05)$, PolyPhen (score $>0.909$ ), Mutation Taster (disease-causing prediction) and Align GVGD (score > C55) have been used as in silico prediction tools to assess the functional effect of the 373 variants.

A list of 12 high risk variants have been selected based on interesting in silico predictions (Table 3) of which seven nonsynonymous variants on $H S D 3 B 1, P B K$, ITIH2, MMS19, PPL, DNAH3 and RASSF2, 1 splicing variation on CFTR, 2 stop-gain variants on CALCOCO2 and LRRC29, 1 frameshift deletion on PABPC3 and 1 frameshift insertion on ZNF677. None of these variants have been listed in the ClinVar database, except CFTRrs1057516216 variant that seems to be "likely pathogenic".

\section{The family specific hypothesis}

We first filtered this list of candidate genes and variants against the additional six BRCAx exome sequenced breast cancer families (BC-TN-F002_BC-TN-F007). All identified variants have been only found in BC-TN-F001, expect the $P A B P C 3$ variant that was found in other Tunisian BRCAx families.

Then, we compared the list of variants identified in this family to results from other WES studies on BRCAx families. Again, variants identified in this study were only found in BC-TN-F001, suggesting a family 


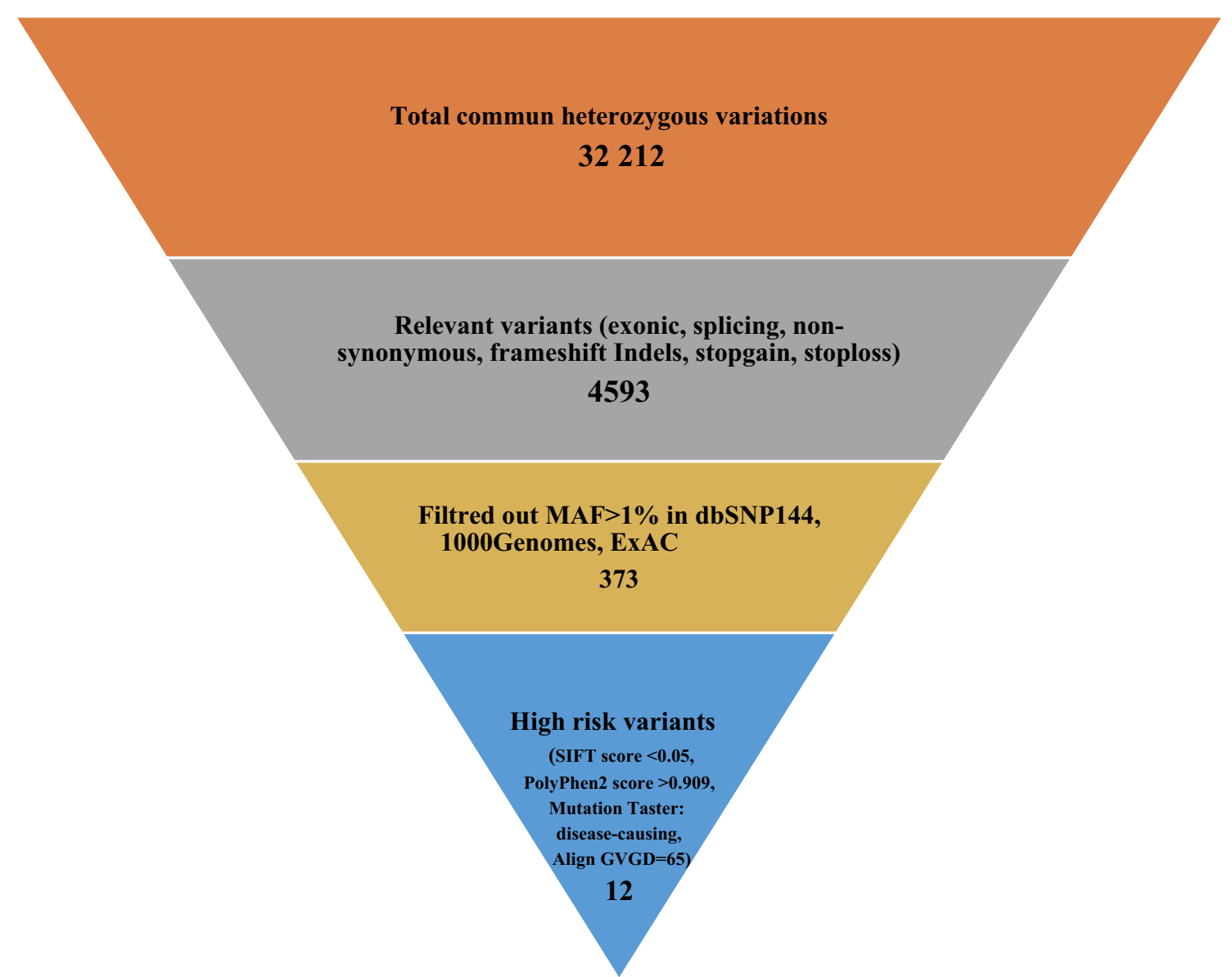

Fig. 2 Number of variants filtered using several criteria determining high risk alleles

specific predisposition to breast cancer. This family specific hypothesis has been suggested to explain the breast cancer predisposition in 4 other WES studies [8, $19-21]$.

We therefore performed a literature curation based on the results of the 4 family specific WES studies and the current one in order to explore this family specific predisposition to breast cancer. Additional file 1: Table S3 summarizes the list of 54 genes identified through these studies as new potential breast cancer candidate genes inherited in a family specific model. We observed that each exome sequenced family showed a specific genetic pattern with a different set of candidate genes. Only $K A T 6 B$ has been reported in two different families from two separate studies $[19,20]$.

In a recent WES study performed on five BRCAx Egyptian families [8], four genes namely LOC100129697, NPIPB1, NBPF10 and PABPC3 have been identified in more than one family. $P A B P C 3$ is also found to be shared between three Egyptian families and the four Tunisian families sequenced in this current study.

\section{Gene set enrichment analysis}

As most of the breast cancer candidate genes identified through family specific predisposition studies lack functional evidence of their involvement in breast carcinogenesis, we pooled the 54 candidate genes identified in separate WES studies (Additional file 1: Table S3) and we performed functional annotation analysis to explore if there is any biological interaction between these genes which may strengthen their association with breast cancer (Additional file1: Table S1; Additional file 2: Figure S1).

Moreover, a comprehensive gene set enrichment combined with a protein-protein interaction analysis was performed using both of EnrichR and Stringdb webtools. Results showed that MMS19 and POLK genes are involved in the DNA repair pathway (Fig. 3). The remaining genes are a part of several pathways involved in cancer etiology such as: Negative regulation of stress activated MAPK cascade (PBK and PINK1), intracellular signal transduction and regulation of autophagosome assembly (LRRK2 and PINK1) and RNA degradation (PABC3 and 


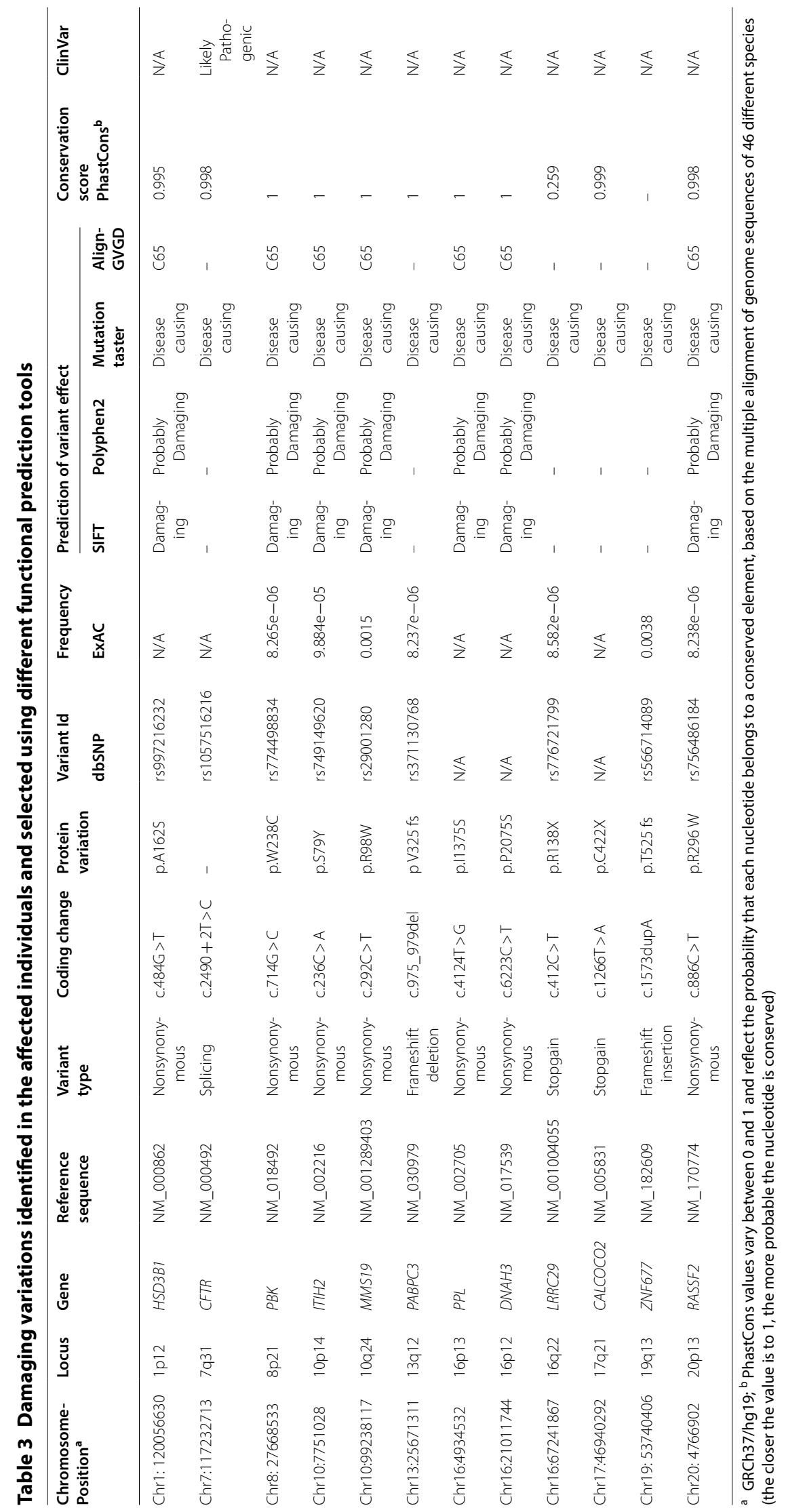




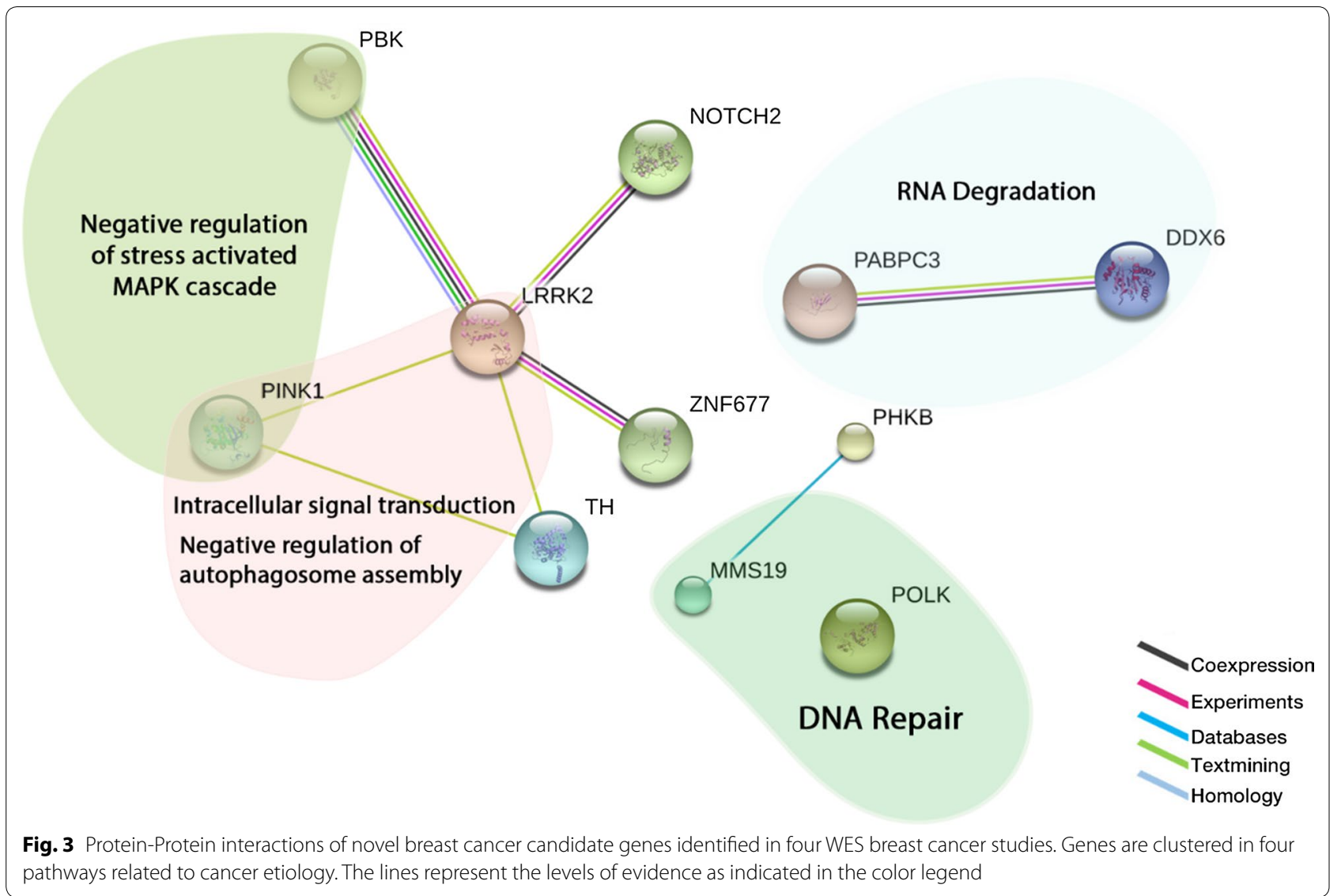

DDX6). NOTCH2 and ZNF677 are highly predicted to be co-expressed with $P B K$ and $L R R K 2$ (Fig. 3).

Finally, we performed a disease genes association analysis using Jensen disease database (PMID: 25484339) by clustering the candidates genes into subgroups involved in a same disease. We, therefore, examined the overlap between these sub-clusters and different cancers namely, breast, ovarian, liver and endometrial cancers (Fig. 4). The results obtained show five top significant genes involved in breast cancers that are DNHA3, KATB6, PDE4DIP, MXRA5 and NBPF10. Of note, NBPF10 is also linked to endometrial cancer and $D N H A 3$ is the only candidate that is involved in all these cancers.

\section{Discussion}

The majority of BRCAx patients with familial breast cancer lack evidence for their genetic predisposition. Multiple models have been proposed to explain the missing heritability. First, recessive and polygenic models of transmission have been proposed to resolve a part of breast cancer remaining heritability [30]. Another class of genetic variations that contributes to familial breast cancer risk includes large deletions and copy number variation [31]. Interactions between

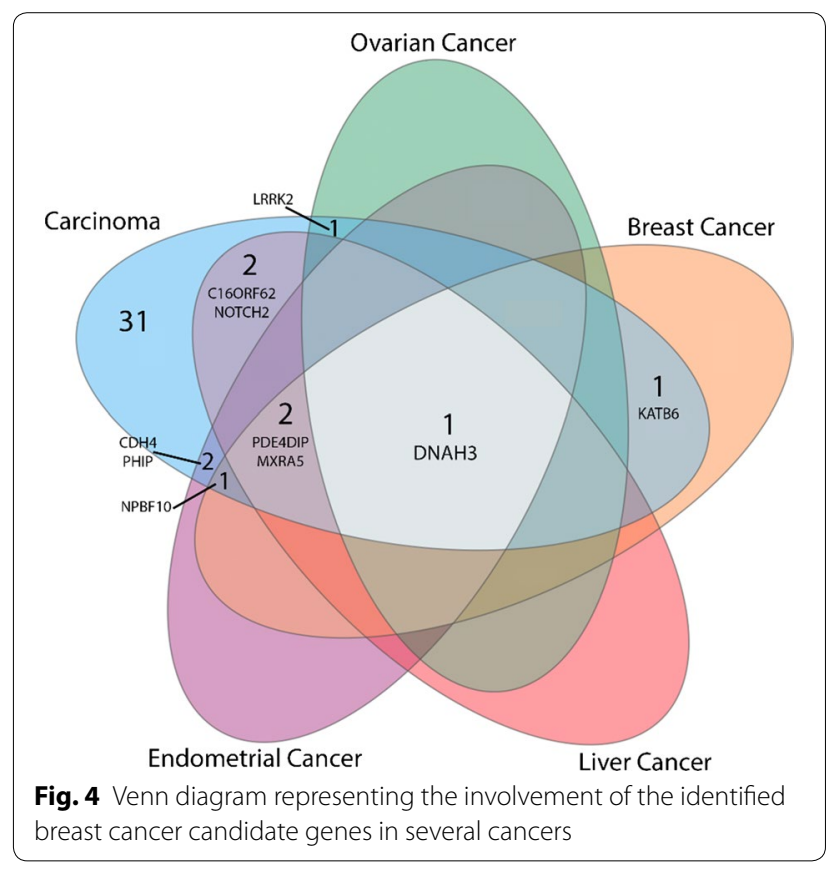

genetic variants and environmental risk factors remain an interesting model to explain breast cancer predisposition in multiple families. However, this model is 
largely unexplored because most of association studies that could address this model are underpowered [32]. Finally, NGS application using family-based approach represents an appropriate modality to identify additional genes with autosomal dominant mechanism of inheritance and thus explains an additional part of the breast cancer familial component [7].

In the present study, two affected sisters from a non BRCA Tunisian breast cancer family have been explored using whole exome sequencing. We excluded unaffected members in our sequenced individuals since they could be non-penetrant carriers.

Thousands of heterozygous variants shared between the two sequenced family members have been identified. However, no deleterious variants have been found within known breast cancer genes. BRCA2-rs4987047 is the only rare exonic variant identified on the known breast cancer susceptibility genes. Despite its potential functional effect [33], the ClinVar predictions classify this variant as benign.

Of note, among 108 exome sequenced families previously reported in 10 breast cancer WES studies, mutations on known breast cancer genes have been reported in only four families because BRCA tests are usually performed before using the whole exome sequencing approach [10-14]. Moreover, the high rate of consanguinity in the Tunisian population, may decrease the prevalence of breast cancer by decreasing the frequency of high penetrant mutations [34].

However, several common variants located on known breast cancer susceptibility genes have been identified in BC-TN-F001 (Table 2). Some of these variants have been previously reported as associated with different cancers as low penetrant polymorphisms. Indeed, two common exonic variants identified on BARD1 gene (rs2229571 and rs1048108) have been identified as low penetrant breast cancer loci in the Chinese population [35]. Moreover, PALB2-rs249954 has been reported to be associated with breast cancer risk [36], CHEK2-rs2236142 is likely associated with a decreased risk of esophageal cancer and lymph node metastasis in a Chinese population [37], $R A D 51 C$-rs12946397 is known to be associated with the risk of head and neck cancer [38] and ATM-rs664143 has been reported to be associated with lung cancer [39]. Given the fact that multiple family members are affected by other cancers such as lung carcinoma and small bowel lymphoma (Fig. 1), the involvement of these variants in this family predisposition to cancer is possible. Therefore, we cannot discard the polygenic model of breast cancer predisposition in this Tunisian breast cancer family.

Despite the fact that these variants have been reported as common low penetrant variants in Caucasians, we cannot estimate their penetrance in the Tunisian population. Indeed, because of different genetic architectures and differences in allele frequencies between populations, variant penetrance may differ from one population to another and a low penetrant variant in one population may be of high penetrance in another population. Further association studies in large Tunisian cohorts are needed to assess the penetrance of these variants in the Tunisian population.

After investigating known breast cancer genes, we explored other genes not yet reported as associated with the breast disease. Twelve high risk variants, predicted as deleterious by four different in silico prediction tools and showing a phenotypic relevance have been selected on the following genes: HSD3B1, CFTR, PBK, ITIH2, MMS19, PABPC3, PPL, DNAH3, LRRC29, CALCOCO2, ZNF677 and RASSF2.

None of the variants identified within these genes have been listed in the ClinVar database, except for the CFTR-rs1057516216 variant that seems to be "likely pathogenic". CFTR (Cystic Fibrosis Transmembrane Conductance Regulator) is a gene that encodes a member of the ATP-binding cassette $(\mathrm{ABC})$ transporter superfamily [40]. Mutations in this gene cause cystic fibrosis, the most common lethal genetic disorder in populations of Northern European descent [41]. However, CFTR is potentially recurrently mutated by chance because of its large size and its involvement in breast carcinogenesis is controversial, thus, it cannot be considered as a potential breast cancer candidate gene. Indeed, it has been proposed that a CFTR mutation may protect against breast cancer [42], however, in another study that correlated the expression level of CFTR and breast cancer histological grading, it was shown that high serum levels of CFTR were associated with a high grade and poorly differentiated tumors [43].

When comparing the identified set of genes with other genes reported in other breast cancer WES studies, we showed that each exome sequenced family has a specific genetic pattern with a different set of candidate genes. Except $P A B P C 3$, genes identified in this Tunisian breast cancer family have not been reported in other breast cancer exome sequenced families, suggesting a family specific genetic predisposition to the disease. $P A B P C 3$ was shared between four Tunisian families and three Egyptian whole exome sequenced families. Moreover, LOC100129697, NPIPB1, NBPF10 have been found in three whole exome sequenced Egyptian families [8]. These genes shared between families from a particular ethnic group (Tunisians and Egyptians) suggest that in populations with high consanguinity and endogamy rates, the ethnic specific breast cancer predisposition model is also plausible. PABPC3 acts in a cytoplasmic regulatory processes of mRNA metabolism [44]. The 
involvement of $P A B P C 3$ in the RNA degradation pathway has been confirmed by the analysis of the biological process and protein-protein networks that we performed in this study (Additional file 2: Figure S1, Fig. 3).

We also showed that the remaining genes are also linked to interesting new pathways such as: negative regulation of stress activated MAPK cascade and intracellular signal transduction and regulation of autophagosome assembly. Only two genes (MMS19 and POLK) are involved in DNA repair pathway, considered as the traditional pathway in which breast cancer genes are involved [45].

MMS19 acts as an adapter between early-acting cytosolic iron-sulfur assembly components and a subset of cellular target iron-sulfur proteins such as ERCC2/XPD, FANCJ and RTEL1, thereby playing a key role in nucleotide excision repair (NER) and RNA polymerase II (POL II) transcription [46]. Of note, the human MMS19 also interacts with estrogen receptors in a ligand-independent manner [47]. POLK is a member of $\mathrm{Y}$ family DNA polymerases, and functions by repairing the replication fork passing through DNA lesions [48]. Recently, POLK have been reported as a new ovarian cancer susceptibility gene [49].

Additional functional annotation analysis using the Jensen disease library, showed that the top significant genes involved in breast cancer are KATB6, PDE4DIP, MXRA5, DNHA3 and NBPF10. KAT6B-a histone acetyl transferase involved in DNA replication, gene expression and regulation, and epigenetic modification of chromosomal structure [50] has been reported as associated with breast cancer in two separate WES studies $[19,20]$.

Consistently with our results, it has been reported that DNHA3 is involved in different cancers including breast cancer [51-53]. DNHA3 (Dynein Axonemal Heavy Chain 3) gene belongs to the dynein family, whose members encode large proteins that are constituents of the microtubule-associated motor protein complex [54]. Among its related pathways we denotes the respiratory electron transport, ATP synthesis by chemiosmosis coupling, and heat production by uncoupling proteins. However, little evidence exist on the roles of PDE4DIP, MXRA5, and NBPF10 in breast carcinogenesis.

In summary, these WES studies results and the functional annotation performed in the present study, altogether showed that MMS19, DNHA3, POLK and KATB6 are interesting breast cancer candidate genes. Variants located on these genes seem to be inherited in a family specific model. PABPC3 seems to be another interesting breast cancer candidate gene that may be associated with breast cancer in an ethnic specific manner as it has been reported in another North African population [8].
Although NGS represents an unprecedented approach to decipher the genetic predisposition to different hereditary diseases, it comes with numerous challenges. Indeed, the different lists of genes that resulted from different breast cancer WES studies may be explained in part by the different pipelines and bioinformatics tools used to analyze these data. In addition, NGS data users apply different filters to help prioritize variants such as the in silico prediction tools that may mis-classify some variants and thus causes erroneous inclusion or exclusion of some variations.

Therefore, in order to assess how much the family specific hypothesis is plausible, we suggest to pool raw data from all breast cancer whole exome sequenced families and re-analyze the resulting data using a common and consensual strategy. Efforts made by the COMPLEXO group in identifying the missing breast cancer heritability via Next generation collaborations represent an excellent initiative to overcome these NGS data analysis challenges [55].

\section{Conclusions}

In the present study we reported a list of new breast cancer candidate genes that seem to be inherited in a family specific and ethnic specific models. Further WES studies on BRCAx Tunisian families and further in vitro or in vivo functional assays are needed to understand their effects and to confirm their association with breast cancer risk. For a better interpretation of NGS data, the scientific community should first overcome NGS data analysis challenges in order to generate more meaningful NGS data and more clinically actionable variants.

\section{Additional files}

Additional file 1: Table S1. Gene set enrichment analysis. Table S2. Summary of SNPs and Indels identified in the 7 BRCAx sequenced Tunisian breast cancer families. Table S3. Putative predisposition family-specific genes in several WES studies using the family-based approach.

Additional file 2: Figure S1. Biological networks and Enriched gene ontology pathways identified by the functional annotation analysis. Enrichment network of the shared candidate disease genes and their upstream regulator based on biological processes using ClueGO Cytoscape plugin. Hyper-geometric (right-handed) enrichment distribution tests, with a p-value significance level of $\leq 0.05$, followed by the Bonferroni adjustment for the terms and leading term groups were selected based on the highest significance. The node size and deeper color indicates greater significance of the enrichment.

\section{Abbreviations}

ABC: ATP-binding cassette; BAM: binary alignment map; BIC: Breast Cancer Information Core; BRCAx: non BRCA; BWA: Burrows-Wheeler Aligner; DNA: DeoxyriboNucleic Acid; GD: Grantham deviation; gDNA: genomic DNA; GV: Grantham variation; INDEL: insertion-deletion; MAF: Minor Allele Frequency; mRNA: Messenger RNA; NER: nucleotide excision repair; NGS: next generation sequencing; PCR: polymerase chain reaction; POL II: RNA polymerase II; RNA: ribonucleic acid; SAM: sequence alignment map; SIFT: Sorting Intolerant From 
Tolerant; SNP: single nucleotide polymorphism; VarAFT: Variant Annotation and Filtering Tool; WES: whole exome sequencing.

\section{Authors' contributions}

Study conception and design: YH and SA. Data acquisition: YH, MB and $\mathrm{CN}$. Analysis and interpretation of data: $\mathrm{YH}, \mathrm{MB}$ and NM have analyzed and interpreted the patient clinic-pathological data. Bioinformatic analysis and networking: $\mathrm{CBH}, \mathrm{KG}$ and $\mathrm{YH}$. Contribution to the interpretation of the results HB, SL, NMJ, HE and ND Technical experiment: MCH, MB, NM. Redaction of the full article: $Y H$. Involvement in the drafting of the manuscript: SA, MB and NM. Critical revision of the article: SA, MSB, RM and OM. Submission procedure: MBR, NM, OM. All authors read and approved the final manuscript.

\section{Author details}

${ }^{1}$ Laboratory of Biomedical Genomics and Oncogenetics, LR16IPT05, Institut Pasteur de Tunis, University of Tunis El Manar, 13, Place Pasteur-BP 74, 1002 Tunis, Tunisia. ${ }^{2}$ Laboratory of Bioinformatics, Biomathematics and Biostatistics, LR16IPT09, Institut Pasteur de Tunis, University of Tunis El Manar, Tunis, Tunisia. ${ }^{3}$ Faculty of Sciences of Bizerte, Carthage University, Tunis, Tunisia. ${ }^{4}$ Department of Medical Oncology, Abderrahmane Mami Hospital, Ariana, Tunisia. ${ }^{5}$ Department of Human Genetics, Charles Nicolle Hospital, Tunis, Tunisia.

\section{Acknowledgements}

The authors are extremely grateful to the patients whose participation made this work possible.

\section{Competing interests}

The authors declare that they have no competing interests.

\section{Availability of data and materials}

All data generated or analyzed during this study are included in this published article and its additional files.

\section{Consent for publication}

Not applicable.

\section{Ethics approval and consent to participate}

Written informed consents were obtained from all participants. Ethical approval according to the Declaration of Helsinki Principles was obtained from the biomedical ethics committee of Institut Pasteur de Tunis (2017/16/E/ hôpital a-m/N1).

\section{Funding}

This work was supported by the Tunisian Ministry of Public Health (PEC4-TUN), the Tunisian Ministry of Higher Education and Scientific Research (LR1 1IPT05 and LR16IPT05) and by the E.C. Grant Agreement No 295097 for FP7 project GM-NCD-Inco.

\section{Publisher's Note}

Springer Nature remains neutral with regard to jurisdictional claims in published maps and institutional affiliations.

Received: 2 March 2018 Accepted: 3 May 2018

Published online: 07 June 2018

\section{References}

1. Rojas K, Stuckey A. Breast cancer epidemiology and risk factors. Clin Obstet Gynecol. 2016;59(4):651-72.

2. Maxwell KN, Nathanson KL. Common breast cancer risk variants in the post-COGS era: a comprehensive review. Breast Cancer Res. 2013;15(6):212

3. Li JJ, et al. Polygenic risk, personality dimensions, and adolescent alcohol use problems: a longitudinal study. J Stud Alcohol Drugs. 2017;78(3):442-51.

4. Shendure J, et al. Advanced sequencing technologies: methods and goals. Nat Rev Genet. 2004;5(5):335.
5. Shendure J, Ji H. Next-generation DNA sequencing. Nat Biotechnol. 2008;26(10):1135.

6. Sokolenko AP, et al. Identification of novel hereditary cancer genes by whole exome sequencing. Cancer Lett. 2015;369(2):274-88.

7. Chandler MR, Bilgili EP, Merner ND. A review of whole-exome sequencing efforts toward hereditary breast cancer susceptibility gene discovery. Hum Mutat. 2016:37(9):835-46.

8. Kim YC, et al. Unique features of germline variation in five Egyptian familial breast cancer families revealed by exome sequencing. PLoS ONE. 2017;12(1):e0167581.

9. Park D, et al. Rare mutations in XRCC2 increase the risk of breast cancer. Am J Hum Genet. 2012;90(4):734-9.

10. Gracia-Aznarez FJ, et al. Whole exome sequencing suggests much of non-BRCA1/BRCA2 familial breast cancer is due to moderate and low penetrance susceptibility alleles. PLOS ONE. 2013;8(2):e55681.

11. Kiiski II, et al. Exome sequencing identifies FANCM as a susceptibility gene for triple-negative breast cancer. Proc Natl Acad Sci. 2014:111(42):15172-7.

12. Park DJ, et al. Rare mutations in RINT1 predispose carriers to breast and Lynch syndrome - spectrum cancers. Cancer Discov. 2014;4(7):804-15.

13. Cybulski $C$, et al. Germline RECQL mutations are associated with breast cancer susceptibility. Nat Genet. 2015;47(6):643.

14. Thompson ER, et al. Exome sequencing identifies rare deleterious mutations in DNA repair genes FANCC and BLM as potential breast cancer susceptibility alleles. PLoS Genet. 2012;8(9):e1002894.

15. Dimassi K, et al. Cancer mortality among reproductive age women in Tunisia. Tunis Med. 2016:94(1):16-22.

16. Corbex M, Bouzbid S, Boffetta P. Features of breast cancer in developing countries, examples from North-Africa. Eur J Cancer. 2014;50(10):1808-18.

17. Al-Eitan LN, Jamous RI, Khasawneh RH. Candidate gene analysis of breast cancer in the Jordanian population of arab descent: a case-control study. Cancer Invest. 2017;35(4):256-70.

18. Bayraktar S, et al. Genotype-phenotype correlations by ethnicity and mutation location in BRCA mutation carriers. Breast J. 2015;21(3):260-7.

19. Wen $\mathrm{H}$, et al. Family-specific, novel, deleterious germline variants provide a rich resource to identify genetic predispositions for BRCAx familial breast cancer. BMC cancer. 2014;14(1):470.

20. Lynch $\mathrm{H}$, et al. Can unknown predisposition in familial breast cancer be family-specific? Breast J. 2013;19(5):520-8.

21. Noh JM, et al. Exome sequencing in a breast cancer family without BRCA mutation. Radiat Oncol J. 2015;33(2):149.

22. Lek $M$, et al. Analysis of protein-coding genetic variation in 60,706 humans. Nature. 2016;536(7616):285.

23. Consortium, G.P. A global reference for human genetic variation. Nature. 2015;526(7571):68.

24. Tavtigian SV, et al. In silico analysis of missense substitutions using sequence-alignment based methods. Hum Mutat. 2008;29(11):1327-36.

25. Chen EY, et al. Enrichr: interactive and collaborative HTML 5 gene list enrichment analysis tool. BMC Bioinform. 2013;14(1):128.

26. Ashburner $M$, et al. Gene ontology: tool for the unification of biology. Nat Genet. 2000;25(1):25

27. Szklarczyk D, et al. STRING v10: protein-protein interaction networks, integrated over the tree of life. Nucleic Acids Res. 2014:43(D1):D447-52.

28. Chen EY, et al. Expression2Kinases: mRNA profiling linked to multiple upstream regulatory layers. Bioinformatics. 2011;28(1):105-11.

29. Shannon P, et al. Cytoscape: a software environment for integrated models of biomolecular interaction networks. Genome Res. 2003;13(11):2498-504.

30. Pharoah PD, et al. Polygenes, risk prediction, and targeted prevention of breast cancer. N Engl J Med. 2008;358(26):2796-803.

31. Enyedi MZ, et al. Simultaneous detection of BRCA mutations and large genomic rearrangements in germline DNA and FFPE tumor samples. Oncotarget. 2016;7(38):61845

32. Nickels $S$, et al. Evidence of gene-environment interactions between common breast cancer susceptibility loci and established environmental risk factors. PLoS Genet. 2013:9(3):e1003284.

33. Johnson N, et al. Counting potentially functional variants in BRCA1, BRCA2 and ATM predicts breast cancer susceptibility. Hum Mol Genet. 2007;16(9):1051-7.

34. Denic S, Bener A. Consanguinity decreases risk of breast cancer-cervical cancer unaffected. Br J Cancer. 2001:85(11):1675. 
35. Liu H, et al. A cross-sectional study of associations between nonsynonymous mutations of the BARD1 gene and breast cancer in Han Chinese women. Asia Pac J Public Health. 2013;25(4_suppl):8S-14S.

36. Chen P, et al. Association of common PALB2 polymorphisms with breast cancer risk: a case-control study. Clin Cancer Res. 2008;14(18):5931-7.

37. $\mathrm{Gu} \mathrm{H}$, et al. Variant allele of CHEK2 is associated with a decreased risk of esophageal cancer lymph node metastasis in a Chinese population. Mol Biol Rep. 2012;39(5):5977-84.

38. Gresner P, et al. Rad51C: a novel suppressor gene modulates the risk of head and neck cancer. Mutat Res Fundam Mol Mech Mutagen. 2014;762:47-54

39. Shen $L$, et al. Association between ATM polymorphisms and cancer risk: a meta-analysis. Mol Biol Rep. 2012;39(5):5719-25.

40. Hyde SC, et al. Structural model of ATP-binding proteing associated with cystic fibrosis, multidrug resistance and bacterial transport. Nature. 1990;346(6282):362.

41. Cutting GR, et al. A cluster of cystic fibrosis mutations in the first nucleotide-binding fold of the cystic fibrosis conductance regulator protein. Nature. 1990;346(6282):366.

42. Li Y, et al. Cystic fibrosis transmembrane conductance regulator gene mutation and lung cancer risk. Lung Cancer. 2010;70(1):14-21.

43. Southey $M C$, et al. CFTR $\triangle F 508$ carrier status, risk of breast cancer before the age of 40 and histological grading in a population-based case-control study. Int J Cancer. 1998;79(5):487-9.

44. Ozturk S, et al. The poly (A)-binding protein genes, EPAB, PABPC1, and PABPC3 are differentially expressed in infertile men with non-obstructive azoospermia. J Assist Reprod Genet. 2016;33(3):335-48.

45. Katsuki Y, Takata M. Defects in homologous recombination repair behind the human diseases: FA and HBOC. Endocr Relat Cancer. 2016;23(10):T19-37
46. Hatfield MD et al. Identification of MMS19 domains with distinct functions in NER and transcription. DNA Repair. 2006;5(8):914-24.

47. Wu X, Li H, Chen JD. The human homologue of the yeast DNA repair and TFIIH regulator MMS19 is an AF-1-specific coactivator of estrogen receptor. J Biol Chem. 2001:276(26):23962-8.

48. Lone $\mathrm{S}$, et al. Human DNA polymerase $\mathrm{k}$ encircles DNA: implications for mismatch extension and lesion bypass. Mol Cell. 2007;25(4):601-14.

49. Stafford $J$, et al. Reanalysis of BRCA1/2 negative high risk ovarian cancer patients reveals novel germline risk loci and insights into missing heritability. PLOS ONE. 2017;12(6):e0178450.

50. Champagne $\mathrm{N}$, et al. Identification of a human histone acetyltransferase related to monocytic leukemia zinc finger protein. J Biol Chem. 1999;274(40):28528-36.

51. Ichikawa T, et al. Immunohistochemical and genetic characteristics of lung cancer mimicking organizing pneumonia. Lung Cancer. 2017:113:134-9.

52. Mclver $L$, et al. Microsatellite genotyping reveals a signature in breast cancer exomes. Breast Cancer Res Treat. 2014;145(3):791-8.

53. Suo C, et al. Integration of somatic mutation, expression and functional data reveals potential driver genes predictive of breast cancer survival. Bioinformatics. 2015;31(16):2607-13.

54. Wickstead B, Gull K. Dyneins across eukaryotes: a comparative genomic analysis. Traffic. 2007;8(12):1708-21.

55. Southey MC, et al. COMPLEXO: identifying the missing heritability of breast cancer via next generation collaboration. Breast Cancer Res. 2013;15(3):402
Ready to submit your research? Choose BMC and benefit from:

- fast, convenient online submission

- thorough peer review by experienced researchers in your field

- rapid publication on acceptance

- support for research data, including large and complex data types

- gold Open Access which fosters wider collaboration and increased citations

- maximum visibility for your research: over $100 \mathrm{M}$ website views per year

At $\mathrm{BMC}$, research is always in progress.

Learn more biomedcentral.com/submissions 